\title{
Coherent intracerebral brain oscillations during learned continuous tracking movements
}

\section{Journal Article}

\section{Author(s):}

Blum, Julia; Lutz, Kai; Pascual-Marqui, Roberto; Murer, Kurt; Jäncke, Lutz

Publication date:

2008-03

Permanent link:

https://doi.org/10.3929/ethz-b-000105767

Rights / license:

In Copyright - Non-Commercial Use Permitted

Originally published in:

Experimental Brain Research 185(3), https://doi.org/10.1007/s00221-007-1164-0 


\title{
Coherent intracerebral brain oscillations during learned continuous tracking movements
}

\author{
Julia Blum • Kai Lutz $\cdot$ Roberto Pascual-Marqui • \\ Kurt Murer · Lutz Jäncke
}

Received: 12 July 2007 / Accepted: 3 October 2007 / Published online: 23 October 2007

(C) Springer-Verlag 2007

\begin{abstract}
The aim of the present study was to assess changes in electroencephalogram (EEG) phase locking between fronto-parietal areas, including the frontal and parietal motor areas, during audiomotor learning of continuous tracking movements. Subjects learned to turn a steering wheel according to a given trajectory in order to minimise the discrepancy between a changing foreground stimulus (controllable by the subjects) and a constant background stimulus. The results of the present study show that increasing practice of continuous tracking movements that are continuously performed in the presence of auditory feedback is not accompanied by decrease in phase locking between areas involved. Moreover, the study confirms that internally produced movements show enhanced coherent activities compared to externally guided movements and therefore suggests that the motor-parietal network is more engaged during internally produced than externally produced movements.
\end{abstract}

\footnotetext{
J. Blum $(\bowtie) \cdot$ K. Lutz · L. Jäncke

Department of Neuropsychology, Institute for Psychology,

University of Zurich, Binzmühlestrasse 14/25,

8050 Zurich, Switzerland

e-mail: j.blum@psychologie.uzh.ch

R. Pascual-Marqui

The KEY Institute for Brain-Mind Research,

University Hospital of Psychiatry, Lenggstrasse 31,

8032 Zurich, Switzerland

\section{K. Murer}

Department of Biology, Institute of Human Movement Sciences and Sport, ETH Zurich, Leonhardstrasse 34,

8092 Zurich, Switzerland
}

\section{Introduction}

Purposeful skilled movements result from orchestrated interactions within a network of brain regions. Extensive transformation and integration processes are especially required for movements guided by external stimuli in order to coordinate current sensory stimuli with upcoming movement commands. To this end, brain regions are assumed to cooperate in a network-like fashion on the basis of synchronized task-specific electric brain activity. Such so-called functional coupling between different brain regions has been studied using coherence and phase locking analysis of EEG (Classen et al. 1998; Gerloff et al. 1998; Manganotti et al. 1998; Lehmann et al. 2006).

Practising a particular motor skill, which is necessary in order to reach an expert level of motor performance, has been proposed to be associated with changes in the strength and pattern of functional coupling (e.g., Serrien and Brown 2002, 2003). Results of a study by Andres et al. (1999) investigating the learning of bimanual movements suggest that enhanced inter-hemispheric sensorimotor coupling was necessary in response to higher task demands in the early training phase of bimanual learning. After subjects reached a proficient level of task performance, inter- and intra-hemispheric coupling decreased again. In addition, Serrien and Brown demonstrated a general increase in coherence in the alpha and beta frequency bands at the beginning of learning a new bimanual task followed by a decrease as task performance improved (Serrien and Brown 2003). Hence, there is evidence that in an over-learned stage, execution of bimanual movements may become less dependent upon the functional coupling between sensorimotor regions. It seems likely that motor programs that are not dependent on sensory input are established during learning. And, it is plausible to assume that movement execution based on recall of 
established motor programs necessitates functional coupling between brain regions to a lesser degree. Whether this idea applies for movements guided by continuously available external information was addressed in a previous study of our group (Blum et al. 2007). In this previous study we employed a tracking task during which movements were guided by continuously available external information. Thus, motor control required ongoing processing and transformation of sensory information into motor commands, leading to ongoing distributed computations within the sensorimotor network. These results emphasized the importance of inter-hemispheric coupling between frontal and parietal regions during early learning of continuous auditory and visual tracking movements. During auditorily guided tracking, a learning-related increase was evident in interhemispheric phase locking between areas of audio-motor integration (inferior parietal lobule) and intra-hemispherically between motor and superior parietal area in the left hemisphere. During visually guided tracking, performance of the task correlated with intra-hemispheric phase locking between the motor and the superior parietal area bilaterally.

Since in that study subjects did not clearly reach a learning plateau, the question remains whether coupling in that fronto-parietal network decreases when the tracking movement is well learned, as has been previously suggested by studies using bimanual coordination learning tasks or learning tasks based on discrete uni- or bimanual movements (Andres et al. 1999; Gerloff and Andres 2002; Serrien and Brown 2003; Serrien et al. 2004). We hypothesize that interregional coupling (e.g., between brain areas associated with motor control) will not change with increasing practice of tracking movements. We therefore anticipate stable coherence measures because sensory motor interactions are necessary, even for the well-learned tracking movements. This finding would certainly be different to those of previous studies that have reported changes in coherence measures obtained for bimanual movements without continuous sensory tracking. In addition, trials with no sensory feedback were embedded as a control condition to examine whether differences in coherence indeed reflect integration of sensory information or, alternatively, reflect some other cortical processes involved in for example establishing an internal movement representation. These non-feedback trials are assumed to solely rely on internal movement representation. Therefore, we hypothesize that the performance of non-feedback trials shows reduced coherence compared with feedback trials.

In the present study, coupling will be primarily assessed by phase locking computations because learning-related changes in our previous study were only detectable when analyzing the phase locking measure (phase component only) but not the coherence measure (amplitude and phase component respected in the interrelation between two signals).

\section{Materials and methods}

Subjects

Sixteen (9 men and 7 women) healthy right-handed volunteers took part in the experiment. Their mean age was $28.3 \pm 5.0$ (SD) years. To measure health a questionnaire regarding neurological health, drug use, hearing and visual deficits was used. Handedness was assessed with the Annett-Handedness-Questionnaire (Annett 1970, 1992). The local ethics committee approved the study and all subjects gave written informed consent.

\section{Experimental design}

In a compensatory motor tracking paradigm, subjects learned to turn a steering wheel according to a given trajectory in order to minimize the discrepancy between a changing foreground stimulus (controllable by the subject) and a constant background stimulus. We attached the steering wheel to a potentiometer in order to measure steering movements between $-125^{\circ}$ to $+125^{\circ}$, with nine bit resolution (512 steps).

The changing foreground stimulus was a train of short sine tones which changed frequency in the range of 400-$500 \mathrm{~Hz}$ in a predefined manner. The background stimulus was a constant sine tone of $2,875 \mathrm{~Hz}$. The stimuli were controlled by commercial experimental software (Presentation, Version 9.81, Neurobehavioral systems, Albany, CA, USA) and presented via commercial headphones (Technics Stereo Headphones RP-F550). The stimulus was built up by 1,000 data points and the refresh rate was set at $60 \mathrm{~Hz}$, resulting in a trial time of $16.67 \mathrm{~s}$. The duration of each foreground stimulus tone was $8 \mathrm{~ms}$, comprising $4 \mathrm{~ms}$ fade-in and $4 \mathrm{~ms}$ fade-out. The intensity of the tone was adjusted to individual preference. Turning the steering wheel to the left decreased the frequency of the foreground tones, whereas turning the steering wheel to the right increased it. When leaving the steering wheel at its position the foreground tone deviated from the constant background tone along a predefined trajectory (Fig. 1). Subjects had to learn to turn the steering wheel so as to keep the pitch of the foreground stimulus and that of the background stimulus as similar as possible. A fixation cross was presented in the centre of a computer screen to avoid eye movements, which contaminate the EEG recording.

Frequencies of the foreground tone were parameterized according to a logarithmic frequency scale with 256 steps. The difference between the foreground stimulus and the background stimulus was continuously calculated by registering the position of the steering wheel at each of the 1,000 data points, forming the sequence of one movement. The difference between the position of the steering wheel and 


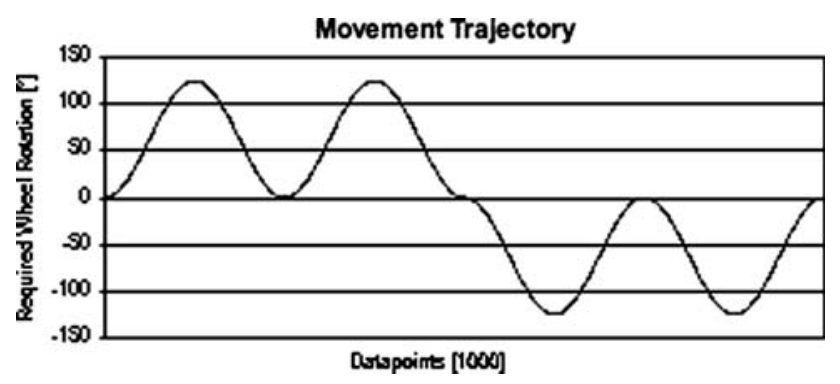

Fig. 1 Movement trajectory. The figure shows the movement trajectory required by the change of the foreground stimulus

the required position given by the target track was taken as a measure of precision of the tracking performance. The subjects were informed about their performance directly after each trial by presenting the deviation from the target track (sum of data point differences between foreground and background stimulus). The single trials were initiated by the subjects themselves, pressing a start button on the steering wheel.

The experimental design consisted of four sessions on subsequent days. In session 1 (pre-training) and 4 (posttraining) EEG was recorded. In the first session subjects were given time to practice the handling of the steering wheel and to become familiar with the principle of the compensatory tracking task. After that, data collection began in a baseline condition (eyes open, eyes closed). The real pattern of the task was subsequently presented twice so that subjects could listen to the changing pattern and acquire a first impression of the required movement. Subjects then performed the task in five subsequent blocks, each of which consisted of seven feedback trials with three intermittent non-feedback trials. During the non-feedback trials only the background stimulus was presented and subjects had to execute the movement without auditory guidance. These trials were indicated by a different colour of the fixation cross. Between each of the blocks, 2 min of baseline condition was recorded (eyes open/eyes closed). Session 4 was similar to session 1 with the exception that the practice condition before data registration was redundant. Sessions 2 and 3 consisted of training sessions without EEG recordings. Subjects performed at least the five blocks of the task. After the two training sessions, subjects should have reached a consistent performance level.

\section{Data acquisition}

The EEG was recorded from 32 scalp electrodes using a Brain Vision amplifier system (BrainProducts, Germany). Silver-silver-chloride-electrodes were used in association with the "Easy Cap System" (FMS Falk Minow Services,
Herrsching-Breitbrunn, Germany) and were placed in the following positions Fp1/2, F3/4, F7/8, Fz, FCz, FT7/8, FC3/4, T7/8, C3/4, Cz, TP7/8, TP9/10, CP3/4, CPz, P7/8, $\mathrm{P} 3 / 4, \mathrm{Pz}, \mathrm{O} 1 / 2$ and Oz. The electro-oculogram (EOG) was recorded from two additional bipolar electrodes placed below the outer canthi of each eye to record horizontal eye movements and above and below the centre of one eye to detect vertical eye movements. The BrainVision Recorder and Analyzer (BrainProducts, Germany) were used to record (electrode impedance $<5 \mathrm{k} \Omega, 0.5-70 \mathrm{~Hz}, 500 \mathrm{sam}$ ples/s) and analyze the data.

EEG data were bandpass filtered from 1 to $50 \mathrm{~Hz}$. In order to correct for eye artefacts (eye blinks, eye movements), an independent component analysis (ICA) algorithm provided in the BrainVision software was used. It has been shown that ICA can effectively detect, separate and remove activity in EEG records from a wide variety of artifactual sources (Jung et al. 1998). In addition, all recorded EEG-epochs were carefully and individually checked for artefacts by visual inspection. The artefact-free EEG material was segmented into the single movements. Each single subject provided 35 feedback trials and 15 non-feedback trials per EEG-session.

\section{Data analysis}

Behavioural data

The steering wheel position was compared with the required target position separately for each of the 1,000 data points, which together form the sequence of one movement, and the deviation for each movement was calculated (Matlab Version 6.5, The MathWorks, Inc., Natick, MA, USA). The mean absolute deviation from target track was used as a performance measure. Each subject provided 35 feedback trials and 15 non-feedback trials. Feedback trials and non-feedback trials were separately checked for outliers (performance $> \pm 2 \mathrm{SD}$ ). These trials were excluded from further analysis for the cortical as well as the behavioural data. Behavioural data were then subjected to a regression analysis. Since subjects who still showed a significant regression after training (post-training condition) did not reach a learning plateau they were excluded from further analysis.

Cortical data

EEG-Data was segmented into epochs of $2 \mathrm{~s}$ duration. These 2-s epochs were then imported into the LORETAKEY software (http://www.unizh.ch/keyinst/loreta). The LORETA method (Pascual-Marqui et al. 1994) computes cortical electric neuronal activity in the form of current 
density, which allows the estimation of intracerebral signals. These signals were then used to calculate the coherence and phase locking between three defined anatomical regions of interest (ROI) in each hemisphere: The first region covers the sensorimotor area, the second the superior and the third the inferior parietal region. The motor ROI was defined using MRIcro (http://www.mricro.com) based on anatomical landmarks, as defined in earlier studies (Roland and Zilles 1996; Fink et al. 1997). The superior and inferior parietal regions were defined using the list of Brodman area information for LORETA voxels provided by the LORETA-KEY software package. The superior parietal ROI included the voxels corresponding to Brodmann area (BA) 7 and the inferior parietal ROI included the voxels corresponding to BA 39 and BA 40. All ROIs were determined in the standardized MNI-space (Evans et al. 1993). In contrast to our previous study, in which the focus was placed on the alpha frequency band, the present study comprises the analysis of both the $\alpha$ - and the $\beta$ - frequency band. Previous studies examining interregional coherence show that the $\alpha$-and the $\beta$-frequency band are valuable in motor tasks. While the $\alpha$-frequency is discussed in terms of general task demands and somatosensory processing, the $\beta$-frequency seems particularly sensitive to motor aspects of the task (Andres et al. 1999; Gerloff et al. 1998; Gerloff and Andres 2002; Serrien and Brown 2002, 2003; Serrien et al. 2003). Therefore, according to the division by the LORETA software, phase locking was calculated in the different frequency bands $\alpha 1: 8.5-10.0 \mathrm{~Hz}, \alpha 2: 10.5-12.0 \mathrm{~Hz}$, $\beta 1: 12.5-18.0 \mathrm{~Hz}, \beta 2: 18.5-21.0 \mathrm{~Hz}, \beta 3: 21.5-30.0 \mathrm{~Hz}$.

To investigate changes related to a late learning phase, EEG-data of the pre-training and the post-training EEG session were compared. EGG data were analyzed by repeated measures ANOVAs with three factors: Condition (feedback trials vs. non-feedback trials); Training (pre-training vs. post -training), and Connection with the following nine levels: inter-hemispheric: motor-motor, BA7-BA7, BA39/ 40-BA39/40; intra-hemispheric: motor-BA7, motor-BA39/ $40, B A 7-B A 39 / 40$, each for the left and the right hemisphere. Additionally, EEG data of the pre-training condition were further analyzed to investigate the early learning effects. The first ten movements of the feedback condition were pooled (phase pre1) and compared with the pooled feedback trials 21-30 (phase pre2). Repeated measures ANOVAs with the following two factors: Phase (prel vs. pre2) and Connection (with the same nine levels as above) were used.

Greenhouse-Geisser correction was applied to guard against effects of heteroscedasticity. The repeated measures ANOVAs were separately computed for the phase locking measure obtained for each frequency band. In case of significant main effects subsequent $t$-tests were conducted for each of the nine levels of the factor Connection, separately examining effects of factor Condition or Training (e.g., Motor-motor, pre-training vs. motor-motor, post-training in feedback trials).

\section{Results}

Behavioural data

Five of the 16 experimental subjects had to be excluded since they still showed a significant regression of performance in the post-training condition and were therefore not considered to have achieved sufficient proficiency. All other subjects showed a stable performance in the posttraining condition (linear regression $p>0.05$ ) and a significant learning effect from pre-training to post-training (two tailed paired sample $t$ test $p<0.05$ ). The mean deviation in the pre-training condition over all valid subjects was $31.2^{\circ}$ and in the post-training condition $12.3^{\circ}$ for the feedback trials and $40.4^{\circ}$ and $21.6^{\circ}$ for the non-feedback trials respectively (see Figs. 2, 3). The mean deviation of feedback trials was significantly different from those of the non-feedback trials in both the pre-training and the post-training condition (two tailed paired sample t-test; pre-training $p<0.01, t=-4.68, d f=10$; post-training $p<0.01 t=-6.11$, $d f=10$ ). However, the learning effect computed as the difference of the mean deviation between pre-training and post-training condition was equal for the feedback $\left(18.9^{\circ}\right)$ and the non-feedback condition $\left(18.8^{\circ}\right)$.

\section{Cortical data}

The analysis of the phase locking data revealed a significant main effect for the factor Condition, but only for the $\beta 3$ frequency band $\left(F(1,10)=6.2, p=0.03, \mathrm{Eta}^{2}=0.38\right)$. Significant interactions between the factors Condition and Training were found for the phase locking measures in $\beta 1$ $\left(F(1,10)=5.5, p=0.04, \mathrm{Eta}^{2}=0.35\right)$ and $\beta 3(F(1,10)=$ $\left.7.3, p=0.02, \mathrm{Eta}^{2}=0.42\right)$. Subsequent $t$-tests revealed that these effects occurred in the comparison of feedback trials with non-feedback trials in phase 2: In general, non-feedback trials showed higher phase locking measures than feedback trials after learning. In the $\beta 1$ frequency band the non-feedback trials showed significant higher phase locking between the inferior and superior parietal region bilaterally compared with those of the feedback trials (left: $p=0.02, t=-2.84, d f=10$; right: $p=0.04, t=-2.35$, $d f=10$ ). Furthermore, phase locking between the motor and the superior parietal region in the right hemisphere was higher in the $\beta 1$ range for non-feedback trials than for feedback trials once learning had taken place $(p=0.05$, $t=-2.28, d f=10$ ). Finally, non-feedback trials of phase 2 showed higher phase locking between the motor and the 
Fig. 2 Learning curve of feedback trials. The figure shows the mean absolute deviation from target track (in degrees) of the 35 feedback trials in the pretraining condition marked in blue and the 35 movements of the post-training condition marked in green. Dashed lines of the respective colour indicate the learning curve \pm standard deviation
Fig. 3 Learning curve of nonfeedback trials. The figure shows the mean absolute deviation from target track (in degrees) of the 15 non-feedback trials in the pre-training condition marked in blue and the 15 movements of the post-training condition marked in green. Dashed lines of the respective colour indicate the learning curve \pm standard deviation
Feedback Trials, Pre-training and Post-training Performance
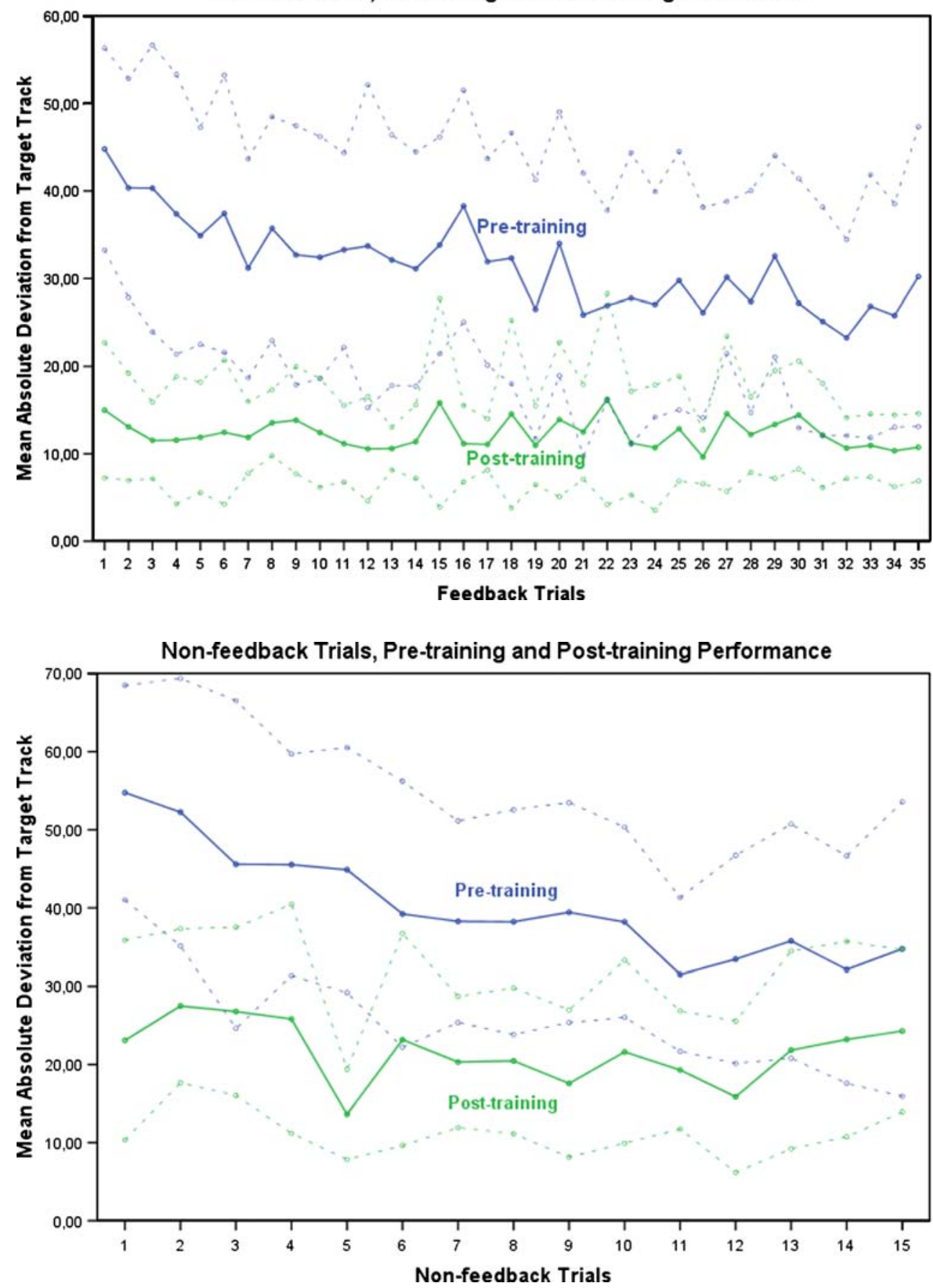

superior parietal region bilaterally than feedback-trials in the range of $\beta 3$ (left: $p=0.03, t=-2.48, d f=10$; right: $p=$ $0.04, t=-2.43, d f=10$ ) (Table 1; Fig. 4).

No significant changes were detected in the range of $\alpha 1$ and $\alpha 2$. No significant changes were observed from the pretraining to post-training condition for the feedback trials or the non-feedback trials.

The analysis of the early learning phase of feedback trials showed a significant interaction between Phase and Connection $\left(F(8,3)=2.6, p=0.00, \mathrm{Eta}^{2}=0.20\right)$ in the range of $\alpha 2$. The subsequen $t$-test showed higher phase locking in the pre 2 condition compared with the pre 1 condition between the motor and superior parietal area $(p=0.03, t=-2.54, d f=10)$ and between the motor and inferior parietal area in the left hemisphere $(p=0.02, t=-2.93, d f=10)$. In the range of $\beta 2$ however the factor Phase was registered on a trend level $\left(F(1,10)=3.4, \quad p=0.1, \quad \operatorname{Eta}^{2}=0.253\right)$. Phase locking decreased between the motor and the superior parietal area in the left hemisphere $(p=0.09, t=1.85, d f=10)$ 
Table 1 Mean phase locking measures of the $\beta 1$ and $\beta 3$ frequency band for the two conditions feedback trials (FB) and non-feedback trials (NFB) after training, the difference between both and the effect size (Cohen's d)

\begin{tabular}{|c|c|c|c|c|}
\hline & FB 2 & NFB 2 & $\mathrm{FB} 2-\mathrm{NFB} 2$ & Cohen's d \\
\hline \multicolumn{5}{|l|}{$\beta 1(12.5-18 \mathrm{~Hz})$} \\
\hline Mo-Mo & $0.794(0.053)$ & $0.798(0.049)$ & $-0.004(0.017)$ & -0.09 \\
\hline Mo-BA7 1 & $0.297(0.071)$ & $0.294(0.071)$ & $0.003(0.023)$ & 0.04 \\
\hline Mo-BA7 r & $0.288(0.055)$ & $0.305(0.050)$ & $-0.017(0.025)$ & -0.33 \\
\hline BA7-BA7 & $0.816(0.071)$ & $0.816(0.070)$ & $0.000(0.009)$ & 0.00 \\
\hline Mo-BA39/40 1 & $0.206(0.065)$ & $0.212(0.056)$ & $-0.006(0.029)$ & -0.10 \\
\hline BA7-BA39/40 1 & $0.365(0.102)$ & $0.386(0.111)$ & $-0.020(0.024)$ & -0.19 \\
\hline Mo-BA39/40 r & $0.200(0.071)$ & $0.201(0.088)$ & $-0.001(0.029)$ & -0.01 \\
\hline BA7-BA39/40 r & $0.380(0.092)$ & $0.391(0.096)$ & $-0.010(0.015)$ & -0.11 \\
\hline BA39/40-BA39/40 & $0.211(0.077)$ & $0.219(0.079)$ & $-0.009(0.014)$ & -0.11 \\
\hline \multicolumn{5}{|l|}{$\beta 3(21.5-30 \mathrm{~Hz})$} \\
\hline Mo-Mo & $0.759(0.055)$ & $0.766(0.064)$ & $-0.006(0.028)$ & -0.11 \\
\hline Mo-BA7 1 & $0.297(0.099)$ & $0.323(0.122)$ & $-0.026(0.034)$ & -0.23 \\
\hline Mo-BA7 r & $0.276(0.083)$ & $0.304(0.102)$ & $-0.029(0.039)$ & -0.31 \\
\hline BA7-BA7 & $0.767(0.057)$ & $0.771(0.058)$ & $-0.004(0.010)$ & -0.07 \\
\hline Mo-BA39/40 1 & $0.266(0.087)$ & $0.280(0.085)$ & $-0.014(0.026)$ & -0.16 \\
\hline BA7-BA39/40 1 & $0.478(0.169)$ & $0.495(0.147)$ & $-0.017(0.041)$ & -0.11 \\
\hline Mo-BA39/40 r & $0.244(0.111)$ & $0.259(0.118)$ & $-0.015(0.026)$ & -0.13 \\
\hline BA7-BA39/40 r & $0.443(0.144)$ & $0.447(0.148)$ & $-0.004(0.024)$ & -0.03 \\
\hline BA39/40-BA39/40 & $0.250(0.126)$ & $0.261(0.122)$ & $-0.011(0.028)$ & -0.09 \\
\hline
\end{tabular}

Standard deviations are given in brackets. $F B 2$ : feedback trials in the post-training, condition, $N F B 2$ : non-feedback trials in the post-training condition, FB2-NFB2 comparison of feedback trials and non-feedback trials, Cohen's $d$ : the difference between the two mean values divided by the pooled standard deviation ( 0.2 indicative of a small, 0.5 of a medium and 0.8 of a large effect size) Connections inter-hemispheric: Mo-Mo $=$ motor areas, $B A 7-B A 7=$ superior parietal areas, $B A 39 / 40=$ inferior parietal areas; intra-hemispheric: $M o-B A 7 \mathbf{l}$ motor-superior parietal area, left hemisphere, $M o-B A 7 r$ motor-superior parietal area, right hemisphere, $M o-B A 39 / 40 l=$ motor-inferior parietal area, left hemisphere, $M o-B A 39 /$ $40 r=$ motor-inferior parietal area, right hemisphere, $B A 7-B A 39 / 40 l=$ superior parietal-inferior parietal area, left hemisphere, $B A 7-B A 39 / 40$ $r=$ superior parietal-inferior parietal area, right hemisphere. Significant $(p<0.05)$ values are given in bold and italics, Trends $(p<0.1)$ in italics

and between the inferior parietal regions $(p=0.06, t=2.10$, $d f=10)$ (Table 2).

\section{Discussion}

Learning effects in the pre-training phase were investigated in order to replicate the findings of our previous study. This complementary analysis of feedback trials in the early learning phase (movement $1-10$ vs. $20-30$ of the pre-training condition) revealed significant increases in phase locking between the motor and the superior parietal and the motor and the inferior parietal region in the left hemisphere. These effects took place in the range of the $\alpha$-frequency band $(\alpha 2)$. This confirms part of the results of our previous study based on the same tracking task (Blum et al. 2007) and corroborates the finding of enhanced coupling during the acquisition phase of the task. In the $\beta$-frequency band ( $\beta 2)$, however, a converse pattern of early learning-related changes was visible (although only marginally significant) with a decrease of phase locking between left-sided motor and superior parietal regions and between the left and the right inferior parietal lobe. According to the literature the increased phase locking in the $\alpha$-frequency band might indicate somatosensory processing while the phase locking in the $\beta$-frequency more strongly indicates control processes associated with executive control aspects related to motor coordination (Andres et al. 1999; Pfurtscheller et al. 1994; Salmelin and Hari 1994; Serrien et al. 2004). Based on these interpretations it is tempting to speculate that somatosensory processing is predominantly operative.

The present study was designed to assess learningrelated changes from an early to a late stage of learning tracking movements that were continuously guided by external stimuli. The hypothesis was that interregional coupling would be stable from an early to a late learning stage. The results show indeed that increasing practice of continuous tracking movements, which are continuously regulated using auditory feedback, is not accompanied by a decrease in phase locking between the involved brain areas. Our findings stand in contrast to those reported by Gerloff and Andres (2002). Their results show that an increase in 


\section{Feeback vs. Non-feedback}

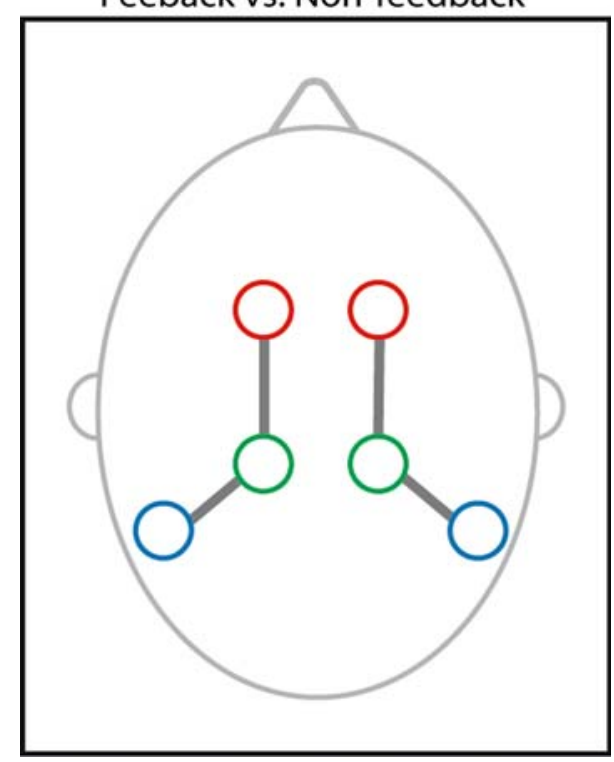

Fig. 4 Feedback vs. non-feedback trials: Solid lines show significant higher phase locking of non-feedback trials compared with feedbacktrials in the post-training condition in the range of $\beta 1$ and $\beta 3$. Motor ROIs in red, superior parietal lobule ROIs in green, inferior parietal lobule ROIs in blue

functional inter-hemispheric coupling between motor areas was only evident during early stages of bimanual co-ordination learning but not during learning of unimanual sequences or during repetition of sequences. Over-learned bimanual movements, on the other hand, revealed a subsequent decrease in inter-hemispheric coupling. These dynamic changes in inter-hemispheric coupling may be attributed to the establishment of motor routines. Our results suggest that the performance of a tracking task guided by continuously available sensory information stays in relation to the sensory stimuli throughout the learning stages. After an initial enhancement of phase locking during the acquisition phase, no further learning-related changes in coupling were detected. This may indicate ongoing somatosensory processing also in later learning stages. Based on the assumption that motor programs reduce the amount of required somatosensory processing, the results may be interpreted as indicating that post-training performance of feedback trials does not depend on recall of motor programs established during training phases. However, it has to be considered that results of coherence computation based on intracerebral model sources may be different from scalp data results (Lehmann et al. 2006). Furthermore, the fact that coupling in the early learning phase was similar for feedback trials and non-feedback trials indicates that the amount of coupling is not due to the processing of external stimuli. Moreover, the coupling does not change from the early (pre-training) to the late learning stage (post-training) even when the movement is executed without sensory guidance (non-feedback trials). Given that performance of movements was stable after training even when executed without sensory guidance, it may be assumed that internal movement representations exist. On a cortical level, however, movement execution does not seem to benefit from established motor programs, which we assumed to be reflected in a decrease of interregional coupling. We therefore suggest that even at a skilled level of performance, imagination and recall of the movement requires coupling within the sensorimotor network. This means that not the engagement of a sensorimotor network by external stimuli is essential for the coherent activity but that purely internally generated movements require also sensory processing and extensive interactions between motor and parietal areas.

In fact and contrary to our assumptions, we found a significant difference in coherent activity after training between externally (feedback trials) and internally guided movements (non-feedback trials). Although the effect size of the differences was only small, the results indicate that engagement of the control centres by external stimuli seems to lead to reduced coherent activity compared with internally generated movements. A study of Gerloff et al. (1998) supports this unexpected result. The authors report higher functional coupling during internally paced finger extensions than during externally paced extensions. The amount of functional coupling is also discussed in relation to task complexity, with enhanced coherent activity during more complex assignments (Gerloff et al. 1998; Manganotti et al. 1998; Serrien and Brown 2002). Therefore, higher complexity of internally guided movements may implicate higher functional coupling within the sensorimotor network during internally guided than during externally guided movements. Alternatively, in respect of the fact that performance in non-feedback trials was poorer that in feedback trials, the differences in coupling may merely be due to the performance difference. This however seems doubtful, as performance differences were already present in the pretraining condition where no differences in phase locking were observed.

In the present study, differences in phase locking between feedback and non-feedback trials occurred in the range of $\beta 1$ and $\beta 3$. In the $\beta 1$-frequency band non-feedback trials showed higher phase locking between the superior and the inferior parietal region bilaterally. These parts can be attributed to the dorsal stream of information processing. Fogassi and Luppino (2005) remark that the visual dorsal stream may be functionally divided and propose that the superior parietal lobule (SPL) is involved in visuomotor transformation processes, whereas the inferior parietal lobule (IPL) is involved in high-level visuomotor representations. They further propose that the IPL in the left hemisphere contributes to the storage of complex representation of actions and the right IPL to perception of spatial 
Table 2 Mean phase locking measures of the $\alpha 2$ and $\beta 2$ frequency band for of the feedback trials 1-10 (pre1) and the feedback trials 21-30 (pre2) of the pre-training conditions, the difference between both and the effect size (Cohen's d)

\begin{tabular}{|c|c|c|c|c|}
\hline & Pre1 & Pre2 & Pre1-Pre2 & Cohen's d \\
\hline \multicolumn{5}{|l|}{$\alpha 2(10.5-12 \mathrm{~Hz})$} \\
\hline Mo-Mo & $0.872(0.049)$ & $0.849(0.082)$ & $0.023(0.042)$ & 0.34 \\
\hline Mo-BA7 1 & $0.343(0.118)$ & $0.378(0.132)$ & $-0.035(0.046)$ & -0.28 \\
\hline Mo-BA7 r & $0.361(0.107)$ & $0.370(0.133)$ & $-0.010(0.053)$ & -0.08 \\
\hline BA7-BA7 & $0.853(0.053)$ & $0.861(0.058)$ & $-0.008(0.026)$ & -0.14 \\
\hline Mo-BA39/40 1 & $0.225(0.043)$ & $0.283(0.074)$ & $-0.057(0.065)$ & -0.94 \\
\hline BA7-BA39/40 1 & $0.369(0.055)$ & $0.386(0.066)$ & $-0.017(0.059)$ & 0.27 \\
\hline Mo-BA39/40 r & $0.262(0.075)$ & $0.259(0.076)$ & $0.003(0.051)$ & 0.04 \\
\hline BA7-BA39/40 r & $0.423(0.124)$ & $0.419(0.1219$ & $0.003(0.067)$ & 0.03 \\
\hline BA39/40-BA39/40 & $0.271(0.073)$ & $0.261(0.076)$ & $0.010(0.066)$ & 0.13 \\
\hline \multicolumn{5}{|l|}{$\beta 2(18.5-21 \mathrm{~Hz})$} \\
\hline Mo-Mo & $0.772(0.054)$ & $0.760(0.068)$ & $0.012(0.042)$ & 0.20 \\
\hline Mo-BA7 1 & $0.310(0.097)$ & $0.260(0.097)$ & $0.050(0.090)$ & 0.52 \\
\hline Mo-BA7 r & $0.311(0.117)$ & $0.271(0.122)$ & $0.040(0.076)$ & 0.33 \\
\hline BA7-BA7 & $0.795(0.058)$ & $0.784(0.078)$ & $0.010(0.044)$ & 0.15 \\
\hline Mo-BA39/40 1 & $0.309(0.107)$ & $0.303(0.093)$ & $0.007(0.139)$ & 0.07 \\
\hline BA7-BA39/40 1 & $0.463(0.093)$ & $0.428(0.120)$ & $0.035(0.104)$ & 0.32 \\
\hline Mo-BA39/40 r & $0.321(0.096)$ & $0.292(0.115)$ & $0.029(0.065)$ & 0.28 \\
\hline BA7-BA39/40 r & $0.425(0.138)$ & $0.399(0.149)$ & $0.026(0.098)$ & 0.18 \\
\hline BA39/40-BA39/40 & $0.240(0.073)$ & $0.213(0.056)$ & $0.027(0.043)$ & 0.42 \\
\hline
\end{tabular}

Standard deviations are given in brackets Pre1: feedback trials 1-10 of the pre-training condition, Pre2: feedback trials 21-30 of the pre-training condition, Pre 1-Pre2: comparison of feedback trials 1-10 and feedback trials 21-30, Cohen's d: the difference between the two mean values divided by the pooled standard deviation ( 0.2 indicative of a small, 0.5 of a medium and 0.8 of a large effect size) Connections inter-hemispheric: $M o-M o=$ motor areas, $B A 7-B A 7=$ superior parietal areas, $B A 39 / 40=$ inferior parietal areas; intra-hemispheric: $M o-B A 7 \mathbf{l}=$ motor-superior parietal area, left hemisphere, $M o-B A 7 r=$ motor-superior parietal area, right hemisphere, $M o-B A 39 / 40 l=$ motor-inferior parietal area, left hemisphere, $M o-B A 39 / 40 r=$ motor-inferior parietal area, right hemisphere, $B A 7-B A 39 / 40 l=$ superior parietal-inferior parietal area, left hemisphere, $B A 7-B A 39 / 40 r=$ superior parietal-inferior parietal area, right hemisphere. Significant $(p<0.05)$ values are given in bold and italics, Trends $(p<0.1)$ in italics

relationships. The involvement of the inferior and the superior parietal lobules in our task is further supported by studies of speech perception that include the investigation of audio-motor integration. Hickok and Poeppel (2000, 2004) propose an auditory ventral and dorsal stream similar to the ventral stream and the dorsal stream of the visual modality. The dorsal auditory stream predominantly in the left hemisphere would be critical for audio-motor integration. Besides some reported lateralization effects, the authors remark that both hemispheres participate in speech perception. In the range of $\beta 3$ we registered enhanced phase locking between the motor region and the superior parietal region bilaterally during non-feedback trials. Interactions between the superior parietal region and the motor region suggest linking of spatial information and movement execution. The difference between feedback and non-feedback trials may therefore reflect greater demands on integrative sensorimotor processes when the movements are not guided by external stimuli.

Significant differences between feedback and non-feedback trials were only registered in the range of the $\beta$-band.
As mentioned above $\beta$-frequency seems more sensitive to motor aspects of coordination. Thus, it can be supposed that significant differences between feedback and non-feedback trials in the late learning stage were related to differences in the processing of motor commands.

\section{Conclusion}

The acquisition phase of tracking movements which are continuously guided by external auditory information is accompanied by an initial increase in interregional coupling. Further practice does not lead to subsequent changes in coupling. The present study therefore supports the suggestion that continuous tracking movements are constantly regulated by sensory feedback, which requires continuous processing of sensory stimuli. This is also true for movements that are well learned. However, the amount of coupling is not due to integration and transformation of external sensory stimuli. The present study demonstrates that performance of internally produced 
movements, which is assumed to solely rely on internal movement representation, also requires extensive interaction between motor and parietal areas. In fact, internally produced movements show enhanced coherent activity within the motor-parietal network compared with externally guided movements. This might be indicative of higher demands on the sensorimotor system during internally guided movements.

\section{References}

Andres FG, Mima T, Schulman AE, Dichgans J, Hallett M, Gerloff C (1999) Functional coupling of human cortical sensorimotor areas during bimanual skill acquisition. Brain 122:855-870

Annett M (1970) A classification of hand preference by association analysis. Br J Psychol 61:303-321

Annett M (1992) Five tests of hand skill. Cortex 28:583-600

Blum J, Lutz K, Jancke L (2007) Coherence and phase locking of intracerebral activation during visuo- and audio-motor learning of continuous tracking movements. Exp Brain Res 182(1):59-69

Classen J, Gerloff C, Honda M, Hallett M (1998) Integrative visuomotor behavior is associated with interregionally coherent oscillations in the human brain. J Neurophysiol 79(3):1567-1573

Evans A, Collins D, Mills S, Brown E, Kelly R, Peters T (1993) 3D statistical neuroanatomical models from 305 MRI volumes. In: Proceedings of IEEE-nuclear science symposium and medical imaging,pp 1813-1817

Fink GR, Frackowiak RS, Pietrzyk U, Passingham RE (1997) Multiple nonprimary motor areas in the human cortex. J Neurophysiol $77: 2164-2174$

Fogassi L, Luppino G (2005) Motor functions of the parietal lobe. Curr Opin Neurobiol 15(6):626-631

Gerloff C, Andres FG (2002) Bimanual coordination and interhemispheric interaction. Acta Psychol (Amst) 110:161-186

Gerloff C, Richard J, Hadley J, Schulman AE, Honda M, Hallett M (1998) Functional coupling and regional activation of human cortical motor areas during simple, internally paced and externally paced finger movements. Brain 121:1513-1531
Hickok G, Poeppel D (2000) Towards a functional neuroanatomy of speech perception. Trends Cogn Sci 4(4):131-138

Hickok G, Poeppel D (2004) Dorsal and ventral streams: a framework for understanding aspects of the functional anatomy of language. Cognition 92(1-2):67-99

Jung TP, Makeig S, Bell AJ, Sejnowski TJ (1998) Independent component analysis of electroencephalographic and event-related potential data. In: Poon P, Brugge J (eds) Auditory processing and neural modeling. Plenum Press, New York, pp 189-197

Lehmann D, Faber PL, Gianotti LR, Kochi K, Pascual-Marqui RD (2006) Coherence and phase locking in the scalp EEG and between LORETA model sources, and microstates as putative mechanisms of brain temporo-spatial functional organization. J Physiol Paris 99(1):29-36

Manganotti P, Gerloff C, Toro C, Katsuta H, Sadato N, Zhuang P et al (1998) Task-related coherence and task-related spectral power changes during sequential finger movements. Electroencephalogr Clin Neurophysiol 109(1):50-62

Pascual-Marqui RD, Michel CM, Lehmann D (1994) Low resolution electromagnetic tomography: a new method for localizing electrical activity in the brain. Int J Psychophysiol 18(1):49-65

Pfurtscheller G, Pregenzer M, Neuper C (1994) Visualization of sensorimotor areas involved in preparation for hand movement based on classification of mu and central beta rhythms in single EEG trials in man. Neurosci Lett 181(1-2):43-46

Roland PE, Zilles K (1996) Functions and structures of the motor cortices in humans. Curr Opin Neurobiol 6:773-781

Salmelin R, Hari R (1994) Spatiotemporal characteristics of sensorimotor neuromagnetic rhythms related to thumb movement. Neuroscience 60(2):537-550

Serrien DJ, Brown P (2002) The functional role of interhemispheric synchronization in the control of bimanual timing tasks. Exp Brain Res 147(2):268-272

Serrien DJ, Brown P (2003) The integration of cortical and behavioural dynamics during initial learning of a motor task. Eur J Neurosci 17:1098-1104

Serrien DJ, Cassidy MJ, Brown P (2003) The importance of the dominant hemisphere in the organization of bimanual movements. Hum Brain Mapp 18(4):296-305

Serrien DJ, Pogosyan AH, Brown P (2004) Cortico-cortical coupling patterns during dual task performance. Exp Brain Res 157(1):79-84 